THE LIE ALGEBRAIC INTERPRETATION OF

THE COMPLETE INTEGRABILITY OF THE ROSOCHATIUS SYSTEM

\author{
Tudor Ratiu*) \\ University of Michigan, Ann Arbor, MI 48109
}

\title{
ABSTRACT
}

The present note answers a question posed by A.G. Reyman [5] as to the Lie algebraic reasons of the complete integrability of a system studied by $E$. Rosochatius [6] .

\section{The Rosochatius System}

Consider the motion of a particle on the sphere $S^{n-1} \subset \mathbb{R}^{n}$ under the influence of the potential

$$
U(x)=\frac{1}{2}\left(A . \check{c} \cdot \underset{\sim}{x}-\|C / x\|^{2}\right)
$$

where $A=\operatorname{diag}\left(a_{1}, \ldots, a_{n}\right), C=\operatorname{diag}\left(c_{1}, \ldots, c_{n}\right)$ and $c / x$ denotes the vector $\left(c_{1} / x_{1}, \ldots, c_{n} / x_{n}\right)$. The equations of motion are

$$
\ddot{x}_{i}=-\partial U / \partial x_{i}+\lambda x_{i}=-\left(a_{i} x_{i}+\frac{c_{i}^{2}}{x_{i}^{3}}\right)+\lambda x_{i}
$$

where $\lambda$ is the Lagrange multiplier defined by the condition that $x \in s^{n-1}$ during the motion. To find $\lambda$, multiply (1.1) by $x_{i}$ and sum over $i$, taking into account that $\|\underset{\sim}{x}\|^{2}=1$, and therefore $x \cdot \underset{\sim}{\dot{x}}=0,\|\underset{\sim}{\dot{x}}\|^{2}+\underset{\sim}{x} \cdot \underset{\sim}{\ddot{x}}=0$. Thus $\lambda=A \underset{\sim}{x} \cdot \underset{\sim}{x}+\|C / \underset{\sim}{x}\|^{2}-\|\underset{\sim}{\dot{x}}\|^{2}$. Set $\underset{\sim}{y}=\underset{\sim}{\dot{x}}$; with $\lambda$ just found $(1.1)$ becomes

$$
\left\{\begin{array}{l}
\dot{x}_{i}=y_{i},\|\|_{\sim}^{x} \|^{2}=1, \underset{v}{x} \cdot \underset{d}{y}=0 \\
\dot{y}_{i}=-a_{i} x_{i}-c_{i} / x_{i}^{3}+\left(A_{\sim}^{x} \cdot \underset{v}{x}+\|c / x\|^{2}-\|y\|^{2}\right) x_{i}
\end{array}\right.
$$

This Hamiltonian system has been shown to be completely integrable by E. Rosochatius [6]. Moser [2] finds a Lax pair for these equations, which however is not equivalent to (1.2) but only implied by it. Moreover, Moser proves that the integrals in involution of (1.2) are eigenvalues of a matrix obtained by a rank two perturbation. Reyman asks what is the Lie algebraic interpretation of Moser's Lax pair, since it does not fit into a general framework developed by him [5].

*) Partially supported by NSF Grant MCS 81-01642 
We shall prove the following: (1.2) is equivalent to a degenerate Euler-Poisson equation [3], [4] on a minimal dimensional orbit of the semidirect product of so(n) with the space of symetric traceless matrices. Introducing a parameter in Moser's Lax pair, it is shown that this Euler-Poisson equation is equivalent to a Hamiltonian system on an invariant submanifold of a subalgebra in the Kac-Moody extension of $s l(n)$. This bihamiltonian formulation of the same problem, yields as usual, the complete integrability of the problem; see also Adler-van Moerbeke [1].

This system is very similar to the C. Neumann system [1], [2], [4], [5], and many results here are implied by facts al ready proved for the Neumann system. What is new, however, is the fact that it seems to be the first completely integrable Euler-Poisson system with non-linear potential - at least to the knowledge of the author.

I would like to thank $H$. Fleschka for drawing my attention to this problem. The exposition that follows is due to length considerations quite dense and hereby based on [4].

\section{The Euler-Poisson Equations}

We start by reviewing a few known facts about orbits in semidirect products.

2.1 Let of be a semisimple Lie algebra with $k$ some constant multiple of the killing form. The semidirect product of $G$ with itself by the adjoint representation is a Lie algebra with underlying vector space of $x$ of and bracket

$$
\left[\left(\xi_{1}, n_{1}\right),\left(\xi_{2}, n_{2}\right)\right]=\left(\left[\xi_{1}, n_{2}\right],\left[\xi_{1}, n_{2}\right]-\left[\xi_{2}, n_{1}\right]\right)
$$

There is a bilinear, symmetric, bi-variant, non-generate two form on this semidirect product induced by $k$ and it is given by

$$
k_{s}\left(\left(\xi_{1}, n_{1}\right),\left(\xi_{2}, n_{2}\right)\right)=k\left(\xi_{1}, n_{2}\right)+k\left(\xi_{2}, n_{1}\right) \text {. }
$$

2.2 In the following considerations, the coadjoint orbit theory plays a central role. If of is any Lie algebra, the KirillovKostant-Souriau theorem states that its coadjoint orbits are symplectic manifolds. If of is semisimple, the equivariant diffeomorphism induced by $k$ makes the adjoint orbits symplectic manifolds. Suppose now that of $=1 V$ with $M$ a Lie subalgebra. Then $N$ * $\approx \xi^{1}(1$ with respect to $k$ ) so that the coadjoint orbits of $\pi^{*}$ can be identified with orbits in $\xi^{1}$ which by the above considerations are symplectic manifolds. Tracing through all the above diffeomorphisms, it can be shown [4] that the Poisson bracket in $\beta^{1}$ is given by 


$$
\{f, g\}(\xi)=-k\left(\left[\pi_{H}(\operatorname{grad} f)(\xi), \pi_{\psi t}(\operatorname{grad} g)(\xi)\right], \xi\right)
$$

for $\xi \in \varepsilon_{z}^{1}, \pi_{\pi}: g \rightarrow \mathfrak{k}$. the comonical projection along $\dot{k}_{z}$ and "grad" the gradient with respect to $k$.

\subsection{Euler-Poisson equations are Hamiltonian systems on adjoint} orbits of the semidirect product $\mathcal{O f} \times \mathcal{g}$ with Hamiltonian of the form $H(\xi, \eta)=\frac{1}{2} k(L \eta, \eta)+V(\xi)$ for $V: G \rightarrow \mathbb{R}$ a smooth function, and $L: g+g$ a linear map. They are of the form

$$
\dot{\xi}=[\xi, L \eta], \quad \dot{\eta}=[\eta, L \eta]+[\xi,(\operatorname{grad} V)(\xi)] ;
$$

see [3] for more information and the connection with reduction. The most famous example of such equations are the equations of the heavy rigid body with a fixed point; in this casecy $=$ so(3) which is identified as Lie algebra with $\mathbb{R}^{3}$ endowed with the crossproduct. In this case $V$ is a linear function of $\xi$. One sees thus, that in general, such Hamiltonian systems are not completely integrable.

$\underline{2.4}$ For $\underset{\sim}{x}, \underset{x}{x} \in \mathbb{R}^{n},\|\underset{\sim}{x}\|=1, \underset{\sim}{x} \cdot \underset{\sim}{y}=0$, set $x=\underset{\sim}{x} \otimes \underset{\sim}{x}-\frac{1}{n}$ Id,

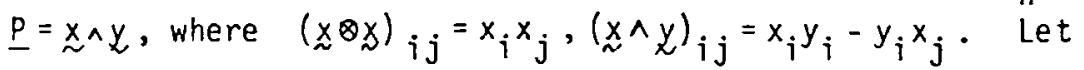
sym denote the vector space of symmetric traceless matrices; then $x \in \operatorname{sym}, \underline{p} \in$ so $(n)$. Split the semidirect product $g=s \ell(n) \times s \ell(n)=$ $=g \oplus \mathbb{Z}, \bar{g}=\operatorname{sym} \times$ so $(n), \mathbb{N}=$ so $(n) \times s y m$. The underlying Lie groups of $O f$ and it respectively are $G=S L(n) \times S \ell(n), N=S O(n) \times \operatorname{sym}$. If $K(A, B)=-\frac{1}{2} \operatorname{Tr}(A B)$, remark that $\xi^{1}=\xi$ where $\perp$ is taken with respect to $k_{s}$. The following has been proved in [4].

2.1 Theorem. The $N$-orbit through $\left(\underset{\sim}{z} \otimes z-\frac{1}{n} I d, 0\right) \in \xi$, $z=(1, \ldots, 1) / \sqrt{n}$, consists of pairs $(X, P) \in-\xi$. With the Kirillov-Kostant-Souriau symplectic form, this $(2 n-2)$-dimensional orbit is symplecticaliy diffeomorphic ia $(X, P) \rightarrow(\underset{\sim}{x}, \underset{\sim}{y})$ to the cotangent bundle $e_{n}$ of $s^{n-1}$ with the symplectic structure induced from $\mathbb{R}^{2 n}$ by $\sum_{i=1} d x_{i} \wedge d y_{i}$. 
2.5 Let us return to the system (1.2). Put $N_{i j}=\frac{c_{i}^{2} x_{j}}{x_{i}^{3}}+\frac{c_{j}^{2} x_{j}}{x_{j}^{3}}$, $N_{i j}=\frac{2 c_{i}^{2}}{x_{i}^{2}}-\frac{2}{n} \sum_{i=1}^{n} \frac{c_{i}^{2}}{x_{i}^{2}}$ and form $N \in$ sym with entries $N_{i j} \cdot$ From now on denote by $A=\operatorname{diag}\left(a_{1}, \ldots, a_{n}\right)-\frac{1}{n}(\operatorname{Tr}(A)) I d \in$ sym .

\subsection{Proposition. The system (1.2) is equivalent to}

$$
\chi=[X, P], \dot{P}=[A+N, X],\|\underset{\sim}{x}\|=1, \underset{\sim}{x} \cdot y=0 .
$$

The proof is a direct, somewhat lengthy, verification.

A comparison between the equations (2.4) and (2.5) shows that (2.5) are degenerate Euler-Poisson equations. Indeed, (2.5) if one chooses $L=$ identity and $V(X)=-K(A, X)+\frac{1}{2} \operatorname{Tr} \phi(X)$, where $\phi(X)$ is chosen such that (grad $V)(X)=-A-N,(2.5)$ becomes a special case of (2.4). The form of $\phi$ is irrelevant; only its existence matters. Specifically, $V(X)$ can be obtained by tracing through the diffeomorphism given by Theorem 2.1 and computing the expression of the push-forward of Rosochatius' potential. We proved the following.

2.3 Theorem The Rosochatius system (1.2) is a Hamiltonian system on the N-orbit in so(n) $x$ sym given by Theorem 2.1. Hamilton's equations coincide with the degenerate Euler-Poisson equations (2.5).

\section{Hamilton's equations in Kac-Moody Lie} algegra setting and complete integrability.

3.1. Introduce the matrices $M \in$ sym, $M_{i j}=\left(c_{i} x_{j} / x_{j}\right)+\left(c_{j} x_{i} / x_{j}\right)$, $M_{i i}=2 c_{i}-2\left(\sum_{i=1}^{n} c_{i}\right) / n, D=\operatorname{diag}\left(c_{1} / x_{i}^{2}, \ldots, c_{n} / x_{n}^{2}\right)-\frac{1}{n}\left(\sum_{i=1}^{n} c_{i} / x_{i}^{2}\right) I d \in \operatorname{sym}$.

3.1 Lemma. If $\|\underset{\sim}{x}\|=1, \underset{\sim}{x} \cdot y=0$, then

$$
[M, X]=[D, X],[D, M]=[N, X]
$$

If $\dot{x}=\mathcal{X}$, then $\dot{M}=[D, P]$.

The proof is a straight forward verification. Using these relations it is easy to show the following. 
3.2 Proposition. If $\|\underset{\sim}{x}\|=1, \underset{\sim}{x} \cdot \underset{\sim}{y}=0$, the degenerate EulerPoisson equations (2.5) are equivalent to

$$
\left(-X+(P+M) \lambda+A \lambda^{2}\right)^{0}=\left[-X+(P+M) \lambda+A \lambda^{2}, P-D+M+A \lambda\right]
$$

If $\lambda=1$, the above Lax equation coincides with the restriction of Moser's to the cotangent bundle of $S^{n-1}$. Equation (3.1) has also a Hamiltonian interpretation, but in the Kirillov-Kostant-Souriau structure of an infinite dimensional Lie algebra.

3.2 Here is a quick review of how (3.1) is Hamiltonian in the $\mathrm{Kac}$-Moody extension of $\mathrm{s} \ell(\mathrm{n})$. Let

$\widetilde{s l(n)}=\left\{\sum_{n \in \mathbb{Z}} \xi_{n} \lambda^{n} \mid\right.$ finite sum $\}$ be the Kac-Moody extension of $s \ell(n)$; the bracket is defined by

$$
\left[\sum_{k \in \mathbb{Z}} \xi_{k} \lambda^{k}, \sum_{n \in \mathbb{Z}} n_{n} \lambda^{n}\right]=\sum_{n \in \mathbb{Z}}\left(\sum_{i+j=n}\left[\xi_{i}, n_{j}\right]\right) \lambda^{n} .
$$

The form $\tilde{\kappa}$ defined by $\tilde{\kappa}\left(\sum_{k \in \mathbb{Z}} \xi_{k} \lambda^{k}, \sum_{n \in \mathbb{Z}} n_{n} \lambda^{n}\right)=\sum_{n+k=-1} k\left(\xi_{k}, n_{n}\right)$, for $\kappa(A, B)=-\frac{1}{2} \operatorname{Tr}(A B), A, B \varepsilon s \ell(n)$ is bilinear, symmetric, bi-invariant and weakly non-degenerate. Let $K=\left\{\sum_{n=0}^{\infty} \xi_{n} \lambda^{n}\right\}$, $N=\left\{\sum_{n=-\infty}^{-1} n_{n} \lambda^{n^{n}}\right\}$; then $\widehat{s \ell(n)}=K \oplus N, K, N$ are Lie subalgebras, $K^{\perp}=K, N^{\perp}=N$. Denote by $\mathscr{G}(\widetilde{s \ell(n))}$ those real values functions on $\widetilde{s l(n)}$ which have gradient with respect to $\tilde{\kappa}$. The submanifold $Q_{A}=\left\{\xi+n \lambda+A \lambda^{2} \mid \xi, n E s l(n)\right\}$ is an invariant submanifold of $K^{\perp}=K$ with respect to the Kirillov-Kostant-Souriau structure given by $G(s \ell(n))$. The submanifold $\left\{-X+(P+M) \lambda+A \lambda^{2}\right\}$ is not invariant but there exists an ad-invariant Hamiltonian whose $\widetilde{K}$-gradient projected onto $K$ equals $P+M-D+A \lambda$. Thus (3.1) is of the form $\dot{\widetilde{\xi}}=\left[\pi_{K}(\operatorname{grad} H) \tilde{\xi}, \tilde{\xi}\right]$, for $\tilde{\xi}=-X+(P+M) \lambda+A \lambda^{2}$ which is the expression of a Hamiltonian vector field on $K^{\perp}$; see [1], [3], [4], [5] for more details and related problems. 
3.3. From (3.1) it follows that the functions $\phi_{k}(x, p)=$

$=\frac{1}{2(k+7)} \operatorname{Tr}\left(-X+(P+M) \lambda+A \lambda^{2}\right)^{k+1}$ are conserved along the flow of (3.1) and thus the coefficients $f_{k}(X, P)$ of $\lambda^{2 k+1}$ in this expansion are conserved along the flow of (2.5).

These functions are in involution. Instead of carrying out here the long computations, we simply remark that the general outline in [4] will work. First, one shows that $\left\{\phi_{K}, \phi_{\ell}\right\}=0$ for every $\lambda$ in the semidirect product $s \ell(n) \times s \ell(n)$ by using the proof of Theorem 3,2 in [4] and a direct computation in which one expresses $M$ as a function of $X$. Specifically, using the expression of the Poisson bracket in the semidirect product for $\phi_{K}, \phi_{l}$, one gets the terms appearing in the proof of Theorem 3.2 of [4] plus three other terms. The first group is handled as in the above mentioned theorem and the last three terms are easily seen to vanish by using the explicit expression of $M$ as a function of $x$. Unfortunately, there is no simple involution theorem at hand which could simplify this step. Thus $\left\{\phi_{K}, \phi_{\ell}\right\}=0$ in the Poisson bracket of $s \ell(n) \times s \ell(n)$ for every $\lambda$. In particular, the highest non-zero $\lambda$-coefficients in this expansion must also vanish, i.e. $\left\{f_{K}, f_{\ell}\right\}=0$ in $s \ell(n) \times s \ell(n)$. Next, one uses Theorem 3.3 of [4] to conclude $\left\{f_{K}, f_{\ell}\right\}=0$ on the N-orbit. This somewhat laborious - but direct - proof shows again how the bihamiltonian formulation of the same problem in Lie algebras is ultimately responsible for involution.

The next step should be the proof of the generic independence of these integrals. We simply refer the reader to [4] for a proof that works - with minor technical modifications - also in this case. Finally, the Kac-Moody Lie algebraic formulations shows that the hyperelliptic curve $\operatorname{det}\left(-X+(P+M) \lambda+A \lambda^{2}-z I d\right)=0$ of genus $n-1$ is isospectral. Applying here the standard methods of Adlervan Moerbeke [1], or Moser [3], the flow linearizes on the Jacobian of this curve.

We proved hence purely Lie algebraically the following.

3.3 Theorem. The Rosochatius system (2.5) is a completely integrable Hamiltonian system with non-linear potential of degenerate EulerPoisson equations on a minimal dimensional orbit of the semidirect product so(n) $x$ sym. Its flow linearizes in the Jacobian of a hyperelliptic curve given by its interpretation as a Hami Ttonian vector field on a submanifold in a subalgebra of the Kac-Moody extension of $s \ell(n)$. 


\section{REFERENCES}

1. M. Adler, P. van Moerbeke, Linearization of Hamiltonian Systems, Jacobi varieties, and Representation Theory, Advances in Math. 38, (1980), 318 - 379.

2. J. Moser, Geometry of Quadrics and Spectral Theory, in "The Chern Sympos ium, Berkeley, June 1979", Springer Verlag (1980), $147-188$.

3. T. Ratiu, Euler-Poisson Equations on Lie Algebras and the N-Dimensional Heavy Rigid Body, Proc. Nat. Acad, Sci. USA, 78, No. 3 (1981), 1327 - 1328.

4. T. Ratiu, The C. Neumann Problem as a Completely Integrable System on an Adjoint Orbit, Trans. Amer. Math. Soc. 264 (1981), $321-329$.

5. A. G. Reyman, Integrable Hamiltonian Systems Connected with Graded Lie Algebras, Zap. Nauk Sem. Leningrad 0.D.T.L., Math. Inst. Steklov (L.0.M.I.), Vol. 95, (1980) 3-54.

6. E. Rosochatius, Über die Bewegung eines Punktes, Inaugural Dissertation, Universität Göttingen, Gebr. Unger, Berlin (1877). 